Running title: Numbers and biological kinematics

\title{
Influence of biological kinematics on abstract concept processing
}

\author{
Arnaud Badets $^{1 *}$, Christel Bidet-Ildei $^{1,2^{*}}$ and Mauro Pesenti ${ }^{3}$
}

${ }^{1}$ Centre de Recherches sur la Cognition et l'Apprentissage, UMR-7295

Centre National de la Recherche Scientifique (CNRS), France

${ }^{2}$ University of Poitiers, France

${ }^{3}$ Institut de Recherches en Sciences Psychologiques et Institute of Neuroscience,

Université catholique de Louvain, Belgium

* Both authors contributed equally to this work.

Corresponding author:

Arnaud Badets

Centre de Recherches sur la Cognition et l'Apprentissage

CNRS, UMR-7295

Maison des Sciences de l'Homme et de la Société

Bât A5; 5, rue Théodore Lefebvre

86000 Poitiers-FRANCE

Tel.: +330632833870

Fax: +330549454616

E-mail: arnaud.badets@univ-poitiers.fr 


\begin{abstract}
During a random number generation task, human beings tend to produce more small numbers than large numbers. However, this small number bias is modulated when motor behaviour, such as a turn of the head, is performed during the random number generation task. This result fits with the finding that number representation is linked to laterally-oriented actions, with small- and large-magnitude numbers generally linked to movement towards the left or the right side of space respectively. To test whether this number-space association is specific to human motor behaviours or extends to any type of laterally oriented movements, we assessed whether the presentation of biological or non-biological leftward or rightward movement affected a subsequent random number generation task. Biological and non-biological movements were obtained by varying the kinematic parameters of the movements. Biological kinematics represented the tangential velocity actually observed in a human pointing movement; non-biological kinematics represented equivalent movements but with an inverse tangential velocity along the path. The results show that only the observation of biological movements induces a space-number bias whereas observing non-biological movements do not. This finding is the first evidence of a link between a biological marker and the semantic representation of a concept as abstract as number.
\end{abstract}

Keywords: number representation, random number generation, biological marker, spacenumber interaction. 


\section{Introduction}

Human beings are highly sensitive to biological movements, and the kinematics of such actions is one of the most important pieces of information to them when observing others (see Blake \& Shiffrar, 2007 for review; Pavlova, 2012). In the seminal paradigm on biological kinematics proposed four decades ago (Johansson, 1973), participants had to identify particular actions from just a small number of light dots placed on the main body joints of a person whom the participants could not see. In this point-light display methodology, only action kinematics characterizing living organisms are retained whereas other types of biological information, such as the form of the limbs, skin texture, or other features of movement, are removed (Atkinson, Dittrich, Gemmell, \& Young, 2004). Observers were able to recognize a human actor walking, running or dancing. Since then, it has been demonstrated that dynamic point-light displays afford sufficient information to identify a person (Cutting \& Kozlowski, 1977) or an arm action (Hill \& Pollick, 2000), the gender of an actor (Kozlowski \& Cutting, 1977), and even their emotions (Atkinson et al., 2004) or intentions (Martel, BidetIldei, \& Coello, 2011). In summary, the human visual system has evolved to recognize biological-based movements, and this sensitivity is largely employed when recognizing or interpreting the behaviours of living organisms.

Interestingly, the existence of a semantic link between biological movements and the processing of abstract meanings like action words or numbers has recently been documented (Andres, Olivier, \& Badets, 2008; Bedny \& Caramazza, 2011 for reviews; Michaux, Pesenti, Badets, Di Luca, \& Andres, 2010). For instance, when the action verb "to run" was presented to participants, the recognition of point-light running behaviour was greatly improved (BidetIldei, Sparrow, \& Coello, 2011). This line of research provides empirical evidence for theories of embodied cognition which hold that sensory-motor mechanisms are co-opted to assist with mental activities whose referents may be distant in time (Wilson, 2002). The co-opted 
mechanisms are thus decoupled from their initial function in bodily experience to support offline conceptual processing (Anderson, 2010). A comparable interaction between biological movements and number processing has also been found: in various tasks and experimental set-ups, small-magnitude numbers were found to be associated with finger grip closing, and large-magnitude numbers with grip opening (Andres, Davare, Pesenti, Olivier, \& Seron, 2004; Andres, Ostry, Nicol, \& Paus, 2008; Badets \& Pesenti, 2010, 2011; Chiou, Wu, Tzeng, Hung, \& Chang, 2012; Lindemann, Abolafia, Girardi, \& Bekkering, 2007). Interestingly, Badets and Pesenti (2011; see also Badets \& Pesenti, 2010; Badets et al. 2012; Loetscher et al. 2008 for head movement) have found an asymmetrical interaction for small number processing. Specifically, they found that the perception of grip closing improved the processing of small numbers only, while no effect was found for the perception of the grip opening movement and large numbers. This bias towards small numbers reveals that the perception of grip closing could evoke grasping for a small object goal.

When asked to generate numbers randomly, participants prefer to produce small rather than large numbers (for a review, see Loetcher \& Brugger, 2007). This is termed the small number bias (SNB) and reflects the fact that, whereas no specific instructions to do so are given, an "internal random generator" biases random generation towards small numbers (Loetcher, Schwarz, Schubiger, \& Brugger, 2008). This natural tendency to produce more small numbers can be modulated by various internal or external cues. For instance, observing pictures mimicking grip closing or opening influences number production (more small numbers than large numbers being produced after observation of a grip closing), whereas observing the closing or opening movements of non-biological geometric shapes has no effect (Badets, Bouquet, Ric, \& Pesenti, 2012). This indicates that the effect does not result from the general processing of motion amplitude. In a same vein, random number generation (RNG) is affected by the performance of (passive whole body motion: Hartmann, Grabherr, \& Mast, 
2012; head turns: e.g., Loetscher \& Brugger, 2007), or observation of (e.g., gaze shifs: Grade, Lefevre, \& Pesenti, 2013) motor actions (Badets et al. 2012). The kinematics of hand movements in space was also found to be affected by number magnitude: when participants had to aim with their hand at a visual target presented to the left or to the right of space after the processing of an Arabic digit, the hand movement was biased towards the left for smallmagnitude numbers and toward the right for large-magnitude numbers (Song \& Nakayama, 2008; see also Wiemers, Bekkering \& Lindemann, in press, for a "motion-arithmetic compatibility" effect wherein the performance in addition and subtraction problem solving was modulated by the direction - upward vs. downward and leftward vs. downward respectively - of hand movements the participants had to perform while calculating). These latter results and the SNB finding have been taken as evidence of the mental number line concept, where small quantities are represented on the left and large quantities on the right side of the line. This number-space association possibly stems from reading and writing habits (Dehaene, 1992).

On the whole, all these findings support the idea that at least part of number semantics rests upon the interaction between the body and its environment, thus providing new evidence supporting embodied cognition theories. These findings indicate that processing a number influences the perception or the production of biological kinematics through a semantic-tomotor link (Badets \& Pesenti, 2010). However, to our knowledge, the possible influence of specific kinematic parameters on numerical semantic processing has never been investigated. In previous studies the stimuli used were either static, or, when human or non-human movements were presented, kinematic parameters were kept constant. Some theories of embodiment proposed that movements that can possibly be self-experienced through body experience are more strongly embodied than others (see Meteyard, Cuadrado, Bahrami \& Vigliocco, 2012 for review). In this context, biological kinematics, which correspond to 
natural human dynamics, are more strongly embodied than movements with non-biological kinematics parameters, which represent movements impossible to reproduce (e.g., when participants have to follow a non-biological elliptic trajectory that does not correspond to the two-third power law, their performance is greatly reduced with an increase in variability and in shape distortions; Viviani, Baud-Bovy \& Redolfi, 1997). Therefore, as our non-biological stimuli used inverse dynamics compatible neither with human movements nor with physical movements in a real environment (indeed, inverse velocity profile do not exist in the physical world and can just be produced artificially in laboratory), a plausible prediction relating to embodied theories of numerical cognition is that number semantics should be more strongly linked to movements with biological kinematic parameters than with non-biological kinematic parameters. The present study explored this prediction thanks to an RNG task coupled with a priming paradigm which used lateralised pointing movements to assess whether and if so how, the SNB was moderated by the type and direction of the movement. Participants were required to randomly produce a number after observing a single dot representing a pointing movement. A pointing movement was used because of its implicit link with numbers through numerosity processing. Indeed, in the context of object counting, pointing and touching gestures are known to help keep track of the count and to match the objects with the verbal string of number names in order to establish one-to-one correspondence. This has been documented in non-human primates who spontaneously exhibit pointing and touching when trained to count an array of items (Boysen, Berntson, Shreyer, \& Hannan, 1995), in human children who count more accurately when they perform pointing and touching actions themselves, compared to when the pointing and touching actions are performed by a puppet manipulated by the experimenter (Alibali \& DiRusso, 1999; Saxe \& Kaplan, 1981), and brain-damaged patients, most of whom (60\% and $65 \%$ respectively) use pointing and touching while counting dots, starting with the leftmost dot and thus implying a left-to-right 
movement (Seron et al., 1991). This has also recently been observed in healthy children and adults, where the direction of pointing during counting related to reading and writing habits (Shaki et al., 2012). Here, two movement conditions were created by varying the kinematics of the movement, thus providing the observers with the same spatial feature information but differing kinematic information: a biological condition used specific biological kinematic features with acceleration and deceleration of the movement during pointing, while the nonbiological condition used inverse biological kinematics. If spatial information is sufficient to induce a space-number bias, possibly by inducing an attentional shift on a mental number line, both biological and non-biological movements should influence number production and moderate the SNB the same way: the proportion of small numbers produced should be higher with a leftward movement and, with a rightward movement, should be lower than (or, at least, not different to) the proportion of large numbers produced, regardless of the type of kinematics. In contrast, if number semantics are only linked to biological kinematics, the moderation of the SNB should only be observed after the biological movement. Moreover, as the probability of producing a number smaller or larger than the previous number differs depending upon the magnitude of that previous number (Costermans, 1990), a further interesting prediction could be made. The influence of movement direction could be measured by the proportional decrease or increase in magnitude of the new number relative to the previous number: a leftward movement might produce a number smaller than the previous one, and a rightward movement might produce a number larger than the previous one. Again, this should be observed in the case of both biological and non-biological kinematics if spatial information is sufficient to induce SNB, but only observed in the case of biological kinematics if it is the result of a specific embodied link.

\section{Method}

\section{Participants}


Sixteen undergraduate students ( 5 female; 2 left-handed, aged between 19 and 22 years, mean $=18.2 \pm 0.7$ years) from the University of Poitiers gave their informed consent to participate in the study for partial course credits. None of them reported any sensory or motor deficits and all had normal or corrected-to-normal vision.

\section{Stimuli and apparatus}

The stimuli were displayed on a 17-inch CRT computer screen (Nyama, spatial resolution: $1024 * 768$ pixels, sampling rate: $60 \mathrm{~Hz}$ ) positioned on a table at a viewing distance of $50 \mathrm{~cm}$. The visual angle of the screen subtended at eye-level was $27.5^{\circ}$ vertical $(\mathrm{V}) * 37^{\circ}$ horizontal $(\mathrm{H})$. Primes were point-light biological or non-biological pointing movements directed to the right or to the left side of space. Each point-light display consisted of a white dot $(97 \mathrm{~cd} / \mathrm{m} 2, \varnothing: 0.6$ of visual angle $)$ presented on a dark background $(0.14 \mathrm{~cd} / \mathrm{m} 2)$. The biological movements came from a previous experiment (Martel et al., 2011) where the spontaneous production by several participants of pointing movements with the forefinger toward a target $1 \mathrm{~cm}$ in diameter placed $10 \mathrm{~cm}$ away in a sagittal plane were recorded with a three dimensional capture system (Zebris ultrasound system, http://www.zebris.de, spatial resolution: $0.5 \mathrm{~mm}$; temporal resolution :100 Hz). It is important to note that with this stimuli configuration, the participants observed the pointing movements as if they were looking at a model in a back position that is as if the movements were performed from their own subjective perspective. Consequently, an action from the model would engage the same effectors in the participants if they were to perform a similar action (see Badets, Blandin, Wright, \& Shea, 2006, and Badets, Blandin, \& Shea, 2006, for a similar observational set-up within different paradigms). From this previous recording, two-dimensional trajectories were reconstructed using Matlab software routines (http://www.mathworks.com/), so as to obtain an avi format movie of $640 * 512$ pixels size with a frame rate of 60 frames/s. At this point, one typical movement was rotated to $20^{\circ}$ and $160^{\circ}$ to obtain a pointing movement directed to 
the right side and the left side respectively. These video sequences represented the biological priming condition insofar as the spatial path and kinematics of the registered dots were similar to the natural movements performed by humans. From these biological point-light displays, we built non-biological equivalent pointing movements with the same trajectory and duration but with modified kinematics along the path using an inverse profile. For this, we inversed the biological pointing kinematics point by point along the path so as to obtain a decrease in tangential velocity followed by an increase of tangential velocity (see Figure 1B). Each priming movement had a duration of $440 \mathrm{~ms}$ and a trajectory length of $9.8 \mathrm{~cm}$. Each priming movement started at $18.5^{\circ}(\mathrm{H}) * 9.1^{\circ}(\mathrm{V})$ and ended at $7.8^{\circ}(\mathrm{H}) * 3.8^{\circ}(\mathrm{V})$ on the left movement and at $28.9^{\circ}(\mathrm{H}) * 3.8^{\circ}(\mathrm{V})$ on the right movement. The imperative stimulus to which the participants responded was a low sound (audio frequency $=1000 \mathrm{~Hz}$, intensity $=2.5$ Watt) played for $150 \mathrm{~ms}$ through computer speakers (multi-media speaker system, frequency response: $22 \mathrm{~Hz} \sim 20 \mathrm{kHz}$, maximum output $=2 * 5$ Watts) placed on each side of the computer screen.

\section{Procedure}

The participants were seated in a dimly lit room in front of the computer screen, with their chest in contact with the table supporting the screen in order to limit their movements. They had to generate numbers between 1 and 10 as randomly as possible (i.e., wholly jumbled sequence, no immediate repetition of the same number, no systematic ascending, descending or somehow ordered sequence, etc.; for a similar method, see Badets et al., 2012) and their responses were paced by a sound (imperative stimulus). Each participant performed three blocks: one training block with no prime to become familiar with the RNG task and two experimental blocks, one with biological and one with non-biological point-light movements to the left or to the right of the screen for half of the stimuli respectively. The order of the two experimental blocks was counterbalanced across participants. In the training block, the 
imperative stimulus was played $750 \mathrm{~ms}$ after the previous response. In the experimental blocks, the imperative stimulus was played after a black screen had been shown for $110 \mathrm{ms,}$ the visual presentation of primes shown for $440 \mathrm{~ms}$ and the black screen for another $50 \mathrm{~ms}$ (see Figure 1). The presentation of primes and stimuli was controlled by E-prime 2.0 (http://www.pstnet.com/); responses were recorded on-line by the experimenter. The experiment comprised 300 trials (60 in training block, $60 * 2$ directions in biological condition, $60 * 2$ directions in non-biological condition; leftward and rightward movements randomly mixed in each block) resulting in a total duration of approximately 30 minutes.

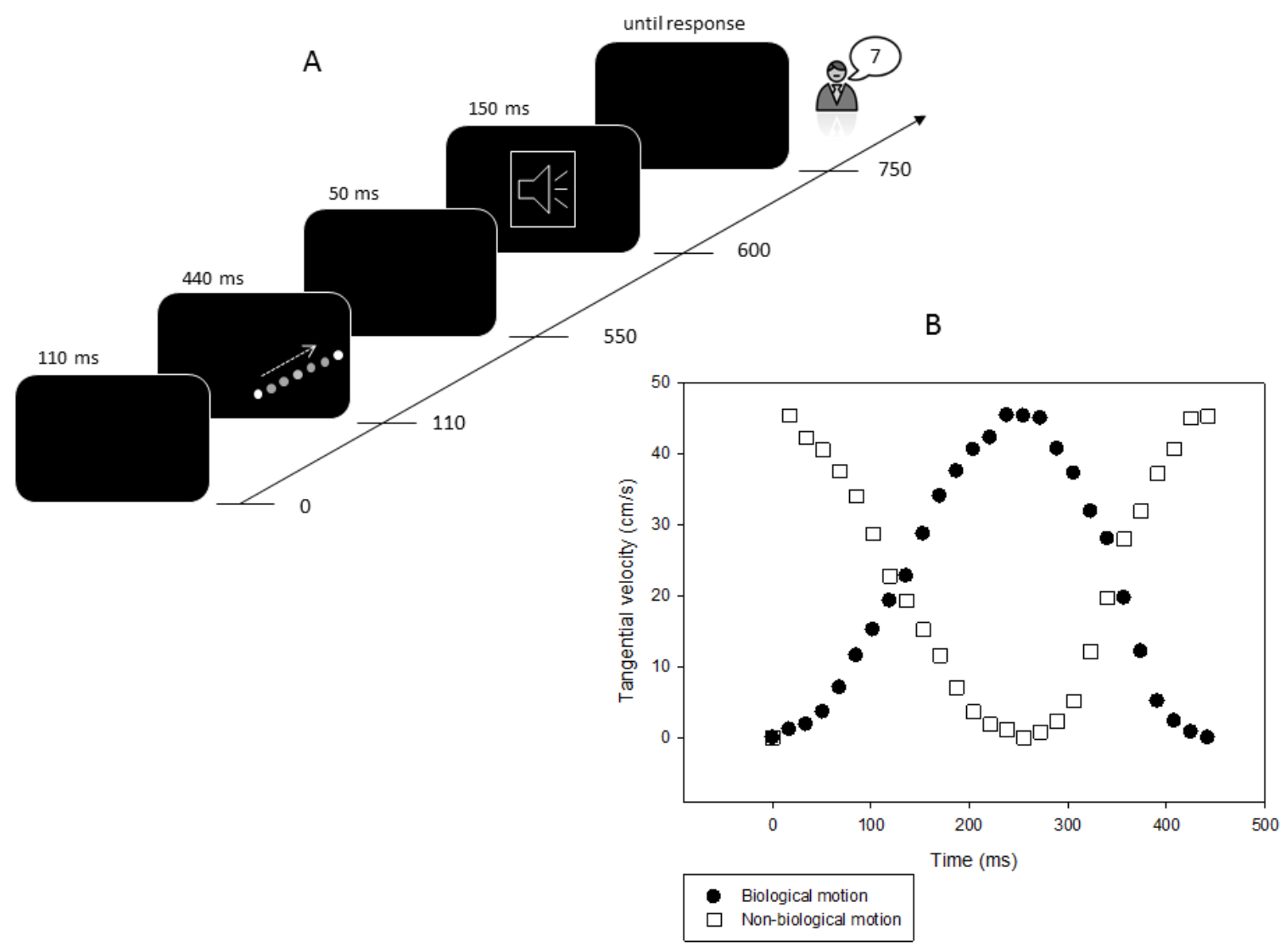

Figure 1: A) Time course of events in the experimental blocks. Visual primes consisted of a biological or non-biological kinematic dot moving toward the left or the right side of the screen. In the figure, the arrow represents the trajectory of the dot. B) Tangential velocity used for the biological (black dots) and the non-biological (white squares) motions. 


\section{Data analysis}

We used the percentage of small numbers (1 to 5) and the percentage increase (see Loetscher et al., 2010 and Grade et al., 2013, for a similar analysis) as dependent variables. For the percentage increase, the data produced after numbers 1 and 10 had been produced (19.6\% of the data) were excluded, because the increase or decrease was totally constrained in these cases. Only the percentage of small numbers and the increase in magnitude relative to the previous number produced were analysed because, as immediate repetitions of the same number were not allowed, small numbers and increase are strictly inversely related to large numbers and decrease, respectively. For each dependent variable, the numbers produced in the training performance were assessed and compared to 50 as the chance level with onetailed Student $t$-tests. For both variables, we presented general results and corrected results. In the corrected results, the percentages obtained in the training condition were used as movement-unbiased baselines and subtracted from those observed in each experimental condition (left and right movements for biological and non-biological primes). Accordingly, the differences obtained represent the pure effect of experimental conditions, independent of the natural tendency to produce small (or large) numbers. An analysis of variance (ANOVA) with prime kinematic (biological vs. non-biological) and prime direction (left vs. right) as within-subject factors was carried out on both general and corrected results. Effect sizes were computed using eta-square estimates. Post-hoc Newman-Keuls comparisons were used to assess the significance of left-right differences in biological and non-biological conditions. Moreover, in the general analysis, each variable (percentage of small number and percentage increase) was compared to chance level (i.e., 50) with one-tailed Student $t$-tests. Where multiple comparisons were performed for the post-tests analyses, a Bonferonni correction was applied. 


\section{Results}

Missed trials (i.e., trials without responses) and errors (e.g., out-of-interval numbers) were excluded from the analyses $(1.84 \%$ of the data).

\section{Percentage of small numbers}

\section{General analysis}

In the training condition, a $t$-test with 50 as the test value revealed that participants produced significantly more small numbers than chance level $\left(\mathrm{M}=52.9 \pm 5.7 \%, t_{15}=2.04\right.$; $p<0.05)$. In the movement conditions, the ANOVA revealed no main effect of prime kinematic (\% small biological: $53.8 \pm 5.4 \%$; non-biological: $52.6 \pm 4.5 \%, F(1,15)<1$ ), but a significant main effect of prime direction $\left(F(1,15)=8.19 ; p<0.05, \eta^{2}=0.4\right)$ with more small numbers produced when movements were directed to the left $(54.8 \pm 4.6 \%)$ than to the right $(51.6 \pm 4.8 \%)$. More importantly, the results revealed a significant interaction between these factors $\left(F(1,15)=7.72 ; p<0.05, \eta^{2}=0.3\right)$. Newman-Keuls post-hoc analyses indicated that the difference between left and right was significant in the biological condition (biological left: $57.6 \pm 5.9 \%$; right: $49.9 \pm 6.8 \%, p<0.05$ ) but not in non-biological condition (non-biological left: $52 \pm 6.2 \%$; right: $53.2 \pm 6.4 \%, p=0.6$ ). Moreover, only the percentage of small numbers in the left biological condition was significantly higher than chance level $\left(t_{15}=5.15 ; p<0.003\right)$.

\section{Corrected analysis}

When the percentage of small numbers produced in the baseline condition was subtracted from the percentages of small numbers produced in the movement conditions, the ANOVA revealed no main effect of prime kinematic $(F(1,15)<1)$, but a significant main effect of prime direction (\% small left: $4.5 \pm 12.7 \%$; right: $-1.7 \pm 13 \% ; F(1,15)=7.98 ; p<$ $\left.0.05 ; \eta^{2}=0.3\right)$ and, critically, a significant interaction between these two factors $(F(1,15)=$ 7.09; $p<0.05 ; \eta^{2}=0.3$; see Figure 2A). Newman-Keuls post-hoc analyses indicated that the 
difference between left and right was significant in the biological condition (left: $M=9.8 \pm$ $14.5 \%$, right: $\mathrm{M}=-4.9 \pm 15.2 \%, p<0.05$ ) but not in the non-biological condition (left: $\mathrm{M}=-0.8$ $\pm 15.2 \%$, right: $\mathrm{M}=1.6 \pm 16.7 \%, p=0.6)$.

\section{Percentage increase}

\section{General analysis}

In the training condition, a $t$-test with 50 as test value revealed that the participants had no specific tendency to produce numbers smaller or larger than the previous number produced $\left(\mathrm{M}=51.4 \% \pm 10.6 \%, t_{15}=0.54 ; p=0.29\right)$. In the movement conditions, the ANOVA revealed no significant main effect of prime kinematics (\% increase biological: $50.7 \pm 5.9 \%$; nonbiological: $49.7 \pm 6.6 \% ; F(1,15)=1.31 ; p=0.27)$, but a significant main effect of prime direction $\left(F(1,15)=5.13 ; p<0.05, \eta^{2}=0.3\right)$ with the rightward movement more frequently leading participants to produce a larger number than the previous number produced (\% increase left: $48.3 \pm 8.01 \%$; right: $52.2 \pm 5.5 \%$ ). More importantly, the results revealed a significant interaction between these factors $\left(F(1,15)=7.84 ; p<0.05, \eta^{2}=0.3\right)$. For this last interaction, post-hoc analyses indicated that the percentage increase was higher when the participants were primed by the biological right movement $(M=54.1 \pm 16.01 \%)$ than by the biological left movement $(M=47.5 \pm 18.7 \%, p<0.01)$, whereas no such difference appeared in the case of the non-biological movement (left: $M=49.2 \pm 8.4 \%$, right: $M=50.2 \pm 6 \%$, $p=0.48$ ). Moreover, only the percentage increase after a right movement in the biological condition was significantly higher than chance level $\left(t_{15}=2.70 ; p<0.04\right)$.

\section{Corrected analysis}

When the percentage increase in the baseline condition was subtracted from the percentage increase in the movement conditions, the ANOVA revealed no significant main 
effect of prime kinematics $(F(1,15)=2.05 ; \mathrm{p}=0.2)$, but it did reveal a significant main effect of prime direction $\left(F(1,15)=5.72 ; p<0.05, \eta^{2}=0.3\right)$ with the rightward movement leading participants to more frequently produce numbers larger than the previous number produced (\% increase difference left: $-4.17 \pm 15 \%$; right: $4.8 \pm 19.6 \%$ ). More importantly, there was a significant interaction between these factors $\left(F(1,15)=6.02 ; p<0.05, \eta^{2}=0.3\right.$; see Figure $\left.2 \mathrm{~B}\right)$. For this last interaction, post-hoc analyses indicated that the percentage increase was higher when the participants were primed by the biological right movement $(\mathrm{M}=8.8 \pm 21 \%)$ than by the biological left movement $(M=-5.5 \pm 19.3 \%, p<0.01)$, whereas no such difference appeared in the case of the non-biological movement (left: $\mathrm{M}=-2.8 \pm 12.2 \%$, right: $\mathrm{M}=0.9 \pm$ $19.6 \%, p=0.26)$.

A

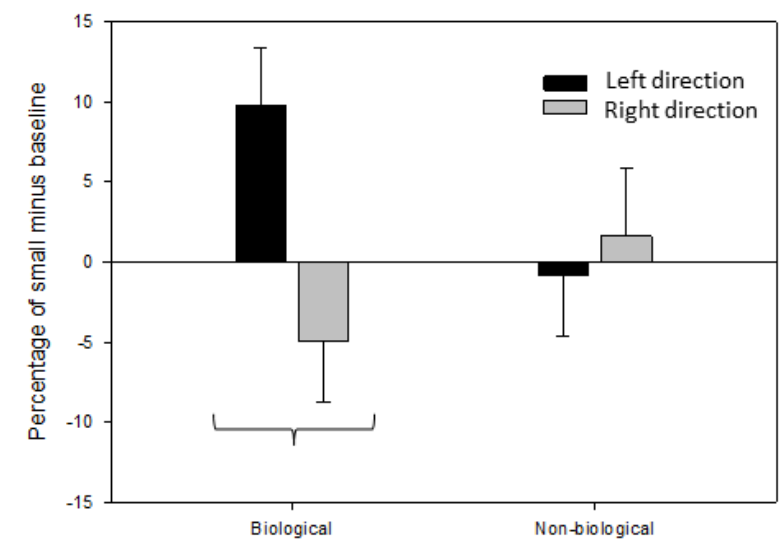

B

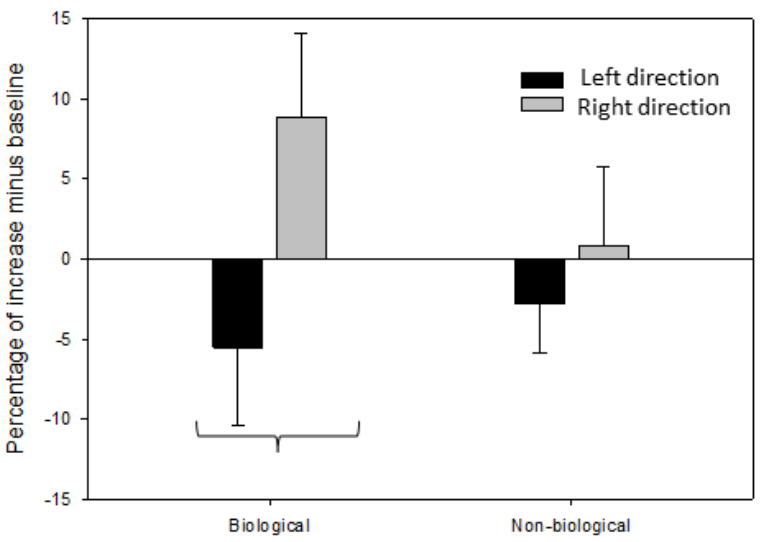

Figure 2: Mean percentage of (A) small numbers and (B) increase as a function of prime type (biological vs. non-biological) and prime direction (left vs. right) when the training condition is used as a baseline. Error bars represent standard errors. Brackets indicate significant difference between directions at $p<0.05$.

\section{Discussion}

This study tested whether or not kinematic biological parameters constitute core information evoking sensorimotor representations (Martel et al., 2011). This was done to 
assess, in the numerical domain, the strength of theories of embodied cognition sustaining that implicit processing of the sensorimotor features of stimuli leads to semantic elaboration (Barsalou, 1999; Glenberg \& Kaschak, 2002). When simply asked to randomly produce numbers, participants generally produced more small numbers than large numbers. That result fits with the so-called SNB. After seeing a dot moving leftward or rightward, they produced more small numbers after a leftward than a rightward movement with biological kinematics, while such a left-right difference was not observed for a movement with non-biological kinematics. This was true even when the productions were corrected for the natural SNB. Importantly, observing a biological movement towards the right side of space led participants to produce numbers larger than the numbers they had produced just before; again, this was not observed with a non-biological movement. In other words, the observation of a pointing movement only influenced the participants' mental selection of a number if the pointing movement had biological kinematics.

Although several studies have previously found a link between human movements and the selection of numbers during an RNG task (Badets et al., 2012; Grade et al., 2013; Hartmann et al., 2012; Loetscher, Bockisch, Nicholls, \& Brugger, 2010; Loetscher, Schwarz, Schubiger, \& Brugger, 2008), the present study is the first to demonstrate that it is the biological nature of the kinematic marker which interacts with the representation of an abstract semantic concept such as numbers. This interaction is better accounted for by the idea of an embodied representation of numbers than by a mere spatial-numerical association stemming from a left-to-right oriented continuum. This is the case because the latter would not predict an effect restricted to biological kinematics but rather an effect that should extend to any kind of lateralised movement, and this was not observed here. In the present context, biological kinematics therefore provides core information that influences semantic access. As suggested by an anonymous reviewer, one might wonder whether this difference between 
biological and non-biological kinematics is not merely due to attentional differences. According to this account, biological kinematics would be simply more salient hence would attract more attention than non-biological ones, which, in turn, would induce a greater effect on number production. Were this to be the case, excluding that differences in the salience and/or the modulation of visual attention drive the observed effect would be needed before interpreting the present finding as an evidence for embodied numerical semantics. However, although interesting, this attentional account appears in fact little plausible when one considers the current state of knowledge concerning biological and non-biological movement processing. Indeed, previous studies that have specifically compared biological and nonbiological movement processing with a single dot showed no response latency difference between the two types of movement (e.g., Bidet-Ildei, Orliaguet, Sokolv \& Pavlova, 2006). This indicates that processing biological and non-biological movements takes the same time, which does not support the idea of a differential involvement of attentional mechanisms. Moreover, when a difference is observed between biological and non-biological stimuli, this difference goes in fact in the direction opposite to the one anticipated from this attentional account: participants are more visually attracted by non-biological than by biological movements. It is, for instance, the case when newborns process biological and non-biological single dot movements (Meary, Kitromilides, Mazens, Graff \& Gentaz, 2007). Finally, this also fits with the classical findings of a greater visual attraction for non-usual than usual stimuli (e.g., Hespos \& Baillargeon, 2001).

Interestingly, the link between biological kinematics and number production appeared more pronounced in the rightward than the leftward movement when the increase relative to the number previously produced is considered. This could be due to some pragmatic aspects of the pointing movement used in our experiment. Indeed, in western cultures, pointing and touching while counting objects displayed in external space usually goes from left to right, 
probably because of reading and writing habits (Shaki et al., 2012). We argue that the effect observed here reflects this preference for left-to-right pointing and touching while counting objects in that the rightward biological movement evoked this object counting gesture hence it induced an increase in magnitude in the verbal counting sequence. By comparison, the leftward biological movement did not evoke such an object counting context. In essence, the non-biological movement does not of course evoke the counting context, and this is the reason why it simply does not moderate the production of numbers in the current set-up.

More broadly, our findings suggest that the biological kinematics of human movement constitute raw information needed to understand action semantics, be it actions observed from the point of view of the observer or of others. It is well known that humans' sensitivity to each other's movements is essential to their social interactions. At a neurophysiological level, this sensitivity is classically related to mirror neurons (i.e., neurons in the premotor cortex that discharge during the observation or the execution of an action; for a review, see Rizzolatti \& Craighero, 2004). Recently, it has been suggested that this mirror system could represent the neuronal basis for the semantic link between numbers and actions (Badets \& Pesenti, 2010). Moreover, given that mirror neurons are viewed as the neuronal substrate onto which communication skills evolve (Rizzolatti \& Arbib, 1998) and in line with previous findings which demonstrate that biological kinematics are also processed through the mirror system (Saygin, Wilson, Hagler, Bates, \& Sereno, 2004; Ulloa \& Pineda, 2007), we can speculate that the sensitivity to biological kinematics could be directly related to human social competencies. However, further investigations are required to examine this theoretical perspective and make firm conclusions.

\section{Conclusion}

Embodied views of numerical cognition suggest a link between number representation and different biological actions (Andres et al. 2004, 2008; Badets \& Pesenti, 2010; 2011; 
Chiou et al. 2012; Grade et al. 2013; Lindemann et al. 2007; Loetscher et al. 2008). The lowest common denominator for these actions is movement kinematics, which represents a key biological signature of human behaviours (Johansson, 1973). In the context of the actionto-semantic relationship, when asked to randomly produce numbers after observing pointlight displays representing biological or non-biological leftward or rightward movements, participants are only affected after the observation of biological movements. This shows that the action perception context and the related increase in the number name string are only evoked by biological kinematics, thus providing critical support to the idea of embodiment of at least some aspects of numerical cognition. 


\section{Acknowledgements}

$\mathrm{AB}$ is a tenured researcher at the National Centre for Scientific Research (CNRS, France). $\mathrm{CBI}$ is associate professor at the University of Poitiers (France). MP is a research associate at the National Fund for Scientific Research (Belgium). 


\section{References}

Alibali, M. W., \& DiRusso, A. A. (1999). The function of gesture in learning to count: More than keeping track. Cognitive Development, 14, 37-55.

Anderson, M. L. (2010). Neural reuse: a fundamental organizational principle of the brain. Behav Brain Sci, 33(4), 245-266; discussion 266-313. doi: 10.1017/S0140525X10000853

Andres, M., Davare, M., Pesenti, M., Olivier, E., \& Seron, X. (2004). Number magnitude and grip aperture interaction. Neuroreport, 15(18), 2773-2777.

Andres, M., Olivier, E., \& Badets, A. (2008). Actions, Words, and Numbers: A Motor Contribution to Semantic Processing? Current Direction in Psychological Science, 17(5), 313-317.

Andres, M., Ostry, D. J., Nicol, F., \& Paus, T. (2008). Time course of number magnitude interference during grasping. Cortex, 44(4), 414-419. doi: 10.1016/j.cortex.2007.08.007

Atkinson, A. P., Dittrich, W. H., Gemmell, A. J., \& Young, A. W. (2004). Emotion perception from dynamic and static body expressions in point-light and full-light displays. Perception, 33(6), 717-746.

Badets, A., Blandin, Y., Wright, D. L., \& Shea, C. H. (2006a). Error detection processes during observational learning. Research Quarterly for Exercise and Sport, 77 (2), 177 84.

Badets, A., Blandin,Y., \& Shea, C. H. (2006b). Intention in motor learning through observation. The Quarterly Journal of Experimental Psychology, 59 (2), 377-386.

Badets, A., Bouquet, C. A., Ric, F., \& Pesenti, M. (2012). Number generation bias after action observation. Experimental Brain Research, 221(1), 43-49. doi: 10.1007/s00221-0123145-1

Badets, A., \& Pesenti, M. (2010). Creating number semantics through finger movement perception. Cognition, 115(1), 46-53.

Badets, A., \& Pesenti, M. (2011). Finger-number interaction: an ideomotor account. Experimental Psychology, 58(4), 287-292. doi: 10.1027/1618-3169/a000095

Barsalou, L. W. (1999). Perceptual symbol systems. Behavioral and Brain Sciences, 22, 577 660.

Bedny, M., \& Caramazza, A. (2011). Perception, action, and word meanings in the human brain: the case from action verbs. Annals of the New York Academy of Science, 1224, 81-95. doi: 10.1111/j.1749-6632.2011.06013.x

Bidet-Ildei, C., Orliaguet, J. P., Sokolv, A. N., \& Pavlova, M. (2006). Perception of elliptic biological motion. Perception, 35 (8), 1137-1147

Bidet-Ildei, C., Sparrow, L., \& Coello, Y. (2011). Reading action word affects the visual perception of biological motion. Acta Psychologica (Amst), 137(3), 330-334. doi: 10.1016/j.actpsy.2011.04.001

Blake, R., \& Shiffrar, M. (2007). Perception of human motion. Annual Review of Psychology, 58, 47-73.

Boysen, S. T., Berntson, G. G., Shreyer, T. A., \& Hannan, M. B. (1995). Indicating acts during counting by a chimpanzee (Pan troglodytes). Journal of Comparative Psychology, 109(1), 47-51.

Chiou, R. Y., Wu, D. H., Tzeng, O. J., Hung, D. L., \& Chang, E. C. (2012). Relative size of numerical magnitude induces a size-contrast effect on the grip scaling of reach-tograsp movements. Cortex, 48(8), 1043-1051. doi: 10.1016/j.cortex.2011.08.001

Costermans, J. (1990). An analysis of the association rates between numbers from 1 to 20 . Cahiers de Psychologie Cognitive 10(1), 3-21. 
Cutting, J. E., \& Kozlowski, L. (1977). Recognizing friends by their walk : Gait perception without falimiarity cues. Bulletin of the Psychonomic Society, 9, 353-356.

Dehaene, S. (1992). Varieties of numerical abilities. Cognition, 44(1-2), 1-42.

Glenberg, A. M., \& Kaschak, M. P. (2002). Grounding language in action. Psychonomic Bulletin Review, 9(3), 558-565.

Grade, S., Lefevre, N., \& Pesenti, M. (2013). Influence of gaze observation on random number generation. Experimental Psychology, 60(2), 122-130. doi: 10.1027/1618$3169 / \mathrm{a} 000178$

Hartmann, M., Grabherr, L., \& Mast, F. W. (2012). Moving along the mental number line: Interactions between whole-body motion and numerical cognition. Journal of Experimental Psychology: Human Perception and Performance, 38(6), 1416-1427. doi: $10.1037 / \mathrm{a} 0026706$.

Hespos, S. J., \& Baillargeon, R. (2001). Reasoning about containment events in very young infants. Cognition, 78(3), 207-245.

Hill, H., \& Pollick, F. E. (2000). Exaggerating temporal differences enhances recognition of individuals from point light displays. Psychological Science, 11(3), 223-228.

Johansson, G. (1973). Visual perception of biological motion and a model for its analysis. Perception \& Psychophysics, 14, 201-211.

Kozlowski, L., \& Cutting, J. E. (1977). Recognizing the sex of a walker from dynamic pointlight displays. Perception \& Psychophysics, 21, 575-580.

Lindemann, O., Abolafia, J. M., Girardi, G., \& Bekkering, H. (2007). Getting a grip on numbers: numerical magnitude priming in object grasping. Journal of Experimental Psychology: Human Perception and Performance, 33(6), 1400-1409. doi: 10.1037/0096-1523.33.6.1400

Loetscher, T., Bockisch, C. J., Nicholls, M. E., \& Brugger, P. (2010). Eye position predicts what number you have in mind. Current Biology, 20(6), R264-265. doi: 10.1016/j.cub.2010.01.015

Loetscher, T., \& Brugger, P. (2007). Exploring number space by random digit generation. Experimental Brain Research, 180(4), 655-665. doi: 10.1007/s00221-007-0889-0

Loetscher, T., Schwarz, U., Schubiger, M., \& Brugger, P. (2008). Head turns bias the brain's internal random generator. Current Biology, 18(2), R60-62. doi: 10.1016/j.cub.2007.11.015

Martel, L., Bidet-Ildei, C., \& Coello, Y. (2011). Anticipating the terminal position of an observed action: Effect of kinematic, structural, and identity information. Visual Cognition, 19(6), 785-798.

Meary, D., Kitromilides, E., Mazens, K., Graff C., \& Gentaz, E. (2007). Four-day-old human neonates look longer at non-biological motions of a single point-of-light. Plos One, 2(1), e186.

Meteyard, L., Cuadrado, S. R., Bahrami, B., \& Vigliocco, G. (2012). Coming of age: a review of embodiment and the neuroscience of semantics. Cortex, 48(7), 788-804. doi: 10.1016/j.cortex.2010.11.002

Michaux, N., Pesenti, M., Badets, A., Di Luca, S., \& Andres, M. (2010). Let us redeploy attention to sensorimotor experience. Behavioral and Brain Sciences, 33(4), 283-284.

Pavlova, M. (2012). Biological motion processing as a hallmark of social cognition. Cerebral Cortex, 22(5), 981-995. doi: 10.1093/cercor/bhr156

Rizzolatti, G., \& Arbib, M. A. (1998). Language within our grasp. Trends Neurosci, 21(5), 188-194.

Rizzolatti, G., \& Craighero, L. (2004). The mirror-neuron system. Annual Review of Neuroscience, 27, 169-192. 
Saxe, G. B., \& Kaplan, R. G. (1981). Gesture in early counting: A developmental analysis. Perceptual and Motor Skills, 53, 513-549.

Saygin, A. P., Wilson, S. M., Hagler, D. J., Jr., Bates, E., \& Sereno, M. I. (2004). Point-light biological motion perception activates human premotor cortex. Journal of Neuroscience, 24(27), 6181-6188.

Seron, X., Deloche, G., Ferrand, I., Cornet, J. A., Frederix, M., \& Hirsbrunner, T. (1991). Dot counting by brain damaged subjects. Brain Cognition, 17(2), 116-137.

Song, J. H., \& Nakayama, K. (2008). Numeric comparison in a visually-guided manual reaching task. Cognition, 106, 994-1003.

Ulloa, E. R., \& Pineda, J. A. (2007). Recognition of point-light biological motion: mu rhythms and mirror neuron activity. Behavioral Brain Research, 183(2), 188-194.

Viviani, P., Baud-Bovy, G., \& Redolfi, M. (1997). Perceiving and tracking kinesthetic stimuli: further evidence of motor-perceptual interactions. Journal of Experimental Psychology: Human Perception and Performance, 23(4), 1232-1252.

Wiemers, M., Bekkering, H., \& Lindemann, O. (in press). Spatial interferences in mental arithmetic: Evidence from the motion-arithmetic compatibility effect. The Quarterly Journal of Experimental Psychology.

Wilson, M. (2002). Six views of embodied cognition. Psychonomic Bulletin Review, 9(4), 625-636. 